\title{
Article
}

\section{Ultrasonic processing of butter oil (Ghee) into oil-in-water emulsions}

\author{
Kulkarni, Chandrashekhar Vishwanath \\ Available at http://clok.uclan.ac.uk/15786/ \\ Kulkarni, Chandrashekhar Vishwanath ORCID: 0000-0002-5621-4791 (2016) \\ Ultrasonic processing of butter oil (Ghee) into oil-in-water emulsions. Journal \\ of Food Processing and Preservation . ISSN 0145-8892
}

It is advisable to refer to the publisher's version if you intend to cite from the work. http://dx.doi.org/10.1111/jfpp. 13170

For more information about UCLan's research in this area go to

http://www.uclan.ac.uk/researchgroups/ and search for <name of research Group>.

For information about Research generally at UCLan please go to http://www.uclan.ac.uk/research/

All outputs in CLoK are protected by Intellectual Property Rights law, including Copyright law. Copyright, IPR and Moral Rights for the works on this site are retained by the individual authors and/or other copyright owners. Terms and conditions for use of this material are defined in the policies page.

\section{CLoK}

Central Lancashire online Knowledge www.clok.uclan.ac.uk

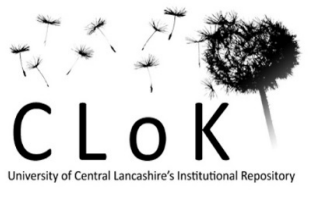




\title{
Ultrasonic Processing of Butter Oil (Ghee) into Oil-in-Water Emulsions
}

\author{
Chandrashekhar V. Kulkarni* \\ Centre for Materials Science, School of Physical Sciences and Computing, University of \\ Central Lancashire, Preston, PR1 2HE, United Kingdom \\ *Corresponding author E-mail: cvkulkarni@uclan.ac.uk, Tel: +44 177289 4339, \\ Fax: +44 1772894981 .
}

\begin{abstract}
Butter oil (ghee) derived from cow or buffalo milk is in semi-solid form at room temperature and contains $>99 \%$ of lipids. Apparent high viscosity and water immiscibility - owing to the hydrophobic nature of lipid components hinder the applicability of butter oil for formulations. Here, we have employed ultrasonic processing technique to create oil-in-water emulsions of butter oil. We show that the butter oil is capable of self-stabilizing an emulsion at low concentration while higher concentrations could be kinetically stabilized using pluronic stabilizer. We were able to modulate water contents of emulsions in the range of $80-95 \%$ and maintain the particle size in submicron range. Reported butter oil emulsions retain hydrophobic properties of the original butter oil within cores of dispersed particles, overcome a problem of butter oil-water immissibility, display fluid consistency and are stable for several months. These properties are highly suitable for developing food, pharmaceutical and cosmetic formulations.
\end{abstract}




\section{Practical Applications}

Ultrasonic processing of butter oil improves its miscibility with water and increase its fluidity thereby enhancing its capability to be used as or to derive formulations for various applications. Further advantage of the reported butter oil emulsions is that they can be loaded with 'polar' in addition to 'non-polar' active molecules, which was hardly possible with formulations merely based on butter oil. This is because an aqueous phase of the butter oil emulsion contains $80-95 \%$ water where polar molecules can easily be solvated. These emulsions, prepared from quite an abundant and economical source, can potentially act as alternatives to the emulsions that usually involve highly expensive lipid components whose extraction or synthesis are rather tough tasks. The ultrasonic processing of butter oil, presented here, could be easily scaled-up using continuous processing modes.

\section{Keywords}

butter oil, ghee, oil-in-water emulsion, butter oil emulsions, lipid based formulations

\section{Introduction}

Butter oil, also called 'ghee' or 'clarified butter' contains $>99 \%$ lipids (USDA, 2016) that are often in the semi-solid form at room temperature (Fatouh et al., 2003). It is usually prepared from a butter generated from cow or buffalo milk (Fatouh et al., 2003; Sharma, 1990; Sujatha and Sarashetti, 2015). As compared to other oils, the butter oil can be advantageous, for instance, for high temperature cooking because it has very high smoking point $\left(\sim 150^{\circ} \mathrm{C}\right)$ (The Culinary Institute of America and Donovan, 1995). Moreover, it has a long shelf life and can be stored at room temperature for 6-8 months (Acharya, 1997; Sserunjogiab et al., 1998). The apparent reason for an extended shelf-life is that it contains very less amount $(\sim 0.5 \%)$ of 
moisture and milk solids - some of which naturally exhibit anti-oxidant properties

(Sserunjogiab et al., 1998) (Fig. 1). Butter, the precursor of the butter oil contains $\sim 18 \%$ moisture (Fig. 1) which is evaporated during the hot $\left(>100^{\circ} \mathrm{C}\right)$ preparation of butter oil whereas $\sim 1 \%$ milk solids are removed by separation techniques (Acharya, 1997).

Ayurveda, a traditional (Indian) medicinal knowledgebase, has referred butter oil as a therapeutic agent (Cope et al., 1996; Heyn, 1987; Pariza, 1991; Sujatha and Sarashetti, 2015; Tirtha, 1998) to treat skin diseases and allergies. Over the years, the butter oil has been used as a suitable medium for preparation of various Ayurvedic - herbal and cosmetic formulations (Bishwas, 2013; Lad, 1999; Liu, 2014; Shah, 2014). It was found to be a superior vehicle for lipophilic herbs as compared to other formulation types such as tablets and powders ((Lad, 1999; Sharma, 1990). However, to extend this application arena further, it is necessary to develop reliable, reproducible and consistent formulations from butter oil. But the difficulties arise due to its highly lipophilic nature. Although butter oil can solubilize lipophilic drugs in it, their efficient delivery is challenged because of the immiscibility with water. One of the effective solutions is believed to be the emulsification of butter oil in aqueous media. Previous reports demonstrate that it is possible to kinetically stabilize butter oil in the form of emulsions using stabilizers such as sodium caseinate (Sangwan et al., 2000), polyvinyl alcohol (Saravanan et al., 2007) and Tween 60 (Newton et al., 2015). In this work we have prepared oil-in-water $(\mathrm{o} / \mathrm{w})$ emulsions of butter oil using a tri-block copolymer - pluronic ${ }^{\circledR}$ F127, which was not used previously for this purpose. This stabilizer has been quite successful for stabilizing a range of lipid based emulsions (Engelskirchen et al., 2011; Nakano et al., 2002; Yaghmur et al., 2005) and hybrid systems developed from them (Kulkarni and Glatter, 2012; Kulkarni et al., 2015; Kulkarni et al., 2011a). These systems have already demonstrated their potential as efficient drug carriers for poorly water soluble 
drugs (Chong et al., 2015). Similarly butter oil emulsions, presented here, are quite promising for formulation applications.

\section{Materials and Methods}

\subsection{Materials}

Unsalted butter ("unsalted English Butter" by Sainsbury’s, UK; Batch 01117011) was purchased from the supermarket and used to prepare butter oil without further treatment. The emulsion stabilizer, triblock copolymer Pluronic ${ }^{\circledR}$ F127 (PEO99PPO67-PEO99) was purchased from Sigma-Aldrich, UK. Water used during entire work was purified using Barnstead Nanopure, Thermoscientific (USA).

\subsection{Preparation of butter oil}

Butter oil was prepared in the following steps: unsalted butter (as mentioned above) was melted on a gentle heat $\left(\sim 70^{\circ} \mathrm{C}\right)$, subsequently the temperature was raised above $\sim 95{ }^{\circ} \mathrm{C}$ to evaporate the moisture (for $\sim 10 \mathrm{~min}$ ) followed by the reduction of the heat down to $\sim 70{ }^{\circ} \mathrm{C}$. The heating was stopped completely when bubbles fade away and solid residues start turning brown. Appearance of characteristic (pleasant) odour signifies that the butter oil is ready. Transparent yellow fluid was then separated from solid residues (brown) by decanting and/or by filtration. This fluid, i.e. the butter oil, was stored at room temperature in its partially solidified form.

\subsection{Preparation of butter oil emulsions}

Butter oil emulsions were prepared using similar protocol as published for lipid emulsions (Kulkarni et al., 2015) but with slightly different parameters. $500 \mathrm{mg}$ of molten butter oil was added to the $20 \mathrm{ml}$ scintillation vial (VWR International Ltd) 
followed by an addition of $9.5 \mathrm{gm}$ of water to make total weight of $10 \mathrm{gm}$. The mixture was then subjected to an ultrasonic processing (Sonics \& Materials Vibra-Cell VCX750, Jencons, UK) for $10 \mathrm{~min}$ at an intermittent pulse of $1 \mathrm{sec}$ with $1 \mathrm{sec}$ delay time using $35 \%$ of the maximum power. Milky white emulsion (containing $5 \mathrm{wt} \%$ butter oil), formed within minutes, was then allowed to cool at room temperature. Emulsions of 10, 15 and $20 \mathrm{wt} \%$ (note: now on wt $\%$ will be designated as \% unless stated differently) of the butter oil were prepared in the same manner as discussed above; corresponding butter oil concentrations were $1000 \mathrm{mg}, 1500 \mathrm{mg}$ and $2000 \mathrm{mg}$ making total weight of $10 \mathrm{gm}$ with water. Emulsion photos were taken by 8.0 megapixel camera from iPhone 5 against white backgrounds.

\subsection{Preparation of butter oil emulsions using pluronic F127 stabilizer}

Mere $0.5 \%$ pluronic F127 stabilizer solution (in water) was sufficient to produce stable emulsions as shown in Fig. 3. These emulsions were prepared using the same ultrasonic processing parameters as mentioned above (section 2.3).

\subsection{Small angle $X$-ray scattering}

Small angle X-ray scattering (SAXS) was utilized to study the nanostructural features of the butter oil and its emulsions. Details of the instrument and the analysis protocol are published earlier (Patil-Sen et al., 2016). The SAXSpace (Anton Paar, Graz, Austria) instrument with $\mathrm{Cu}-\mathrm{K}_{\alpha}$ radiation at a wavelength $(\lambda)$ of $0.154 \mathrm{~nm}$ was used in the high resolution mode to detect a minimum scattering vector, $q_{\min }$, of $0.04 \mathrm{~nm}^{-1}$ ( $q=$ $(4 \pi / \lambda) \sin \theta$, where $2 \theta$ is the scattering angle). Samples were prepared in $1 \mathrm{~mm}$ quartz cell (Anton Paar, Graz, Austria) and studied under vacuum and temperature $(25 \pm 0.1$ $\left.{ }^{\circ} \mathrm{C}\right)$ controlled environment. Three images of typical exposure times of $300 \mathrm{sec}$ were 
averaged to obtain $1 \mathrm{D}$ scattering profiles which were then analysed using The SAXStreat software (Anton Paar, Graz, Austria) (Patil-Sen et al., 2016).

\subsection{Particle size by Mastersizer}

The particle size and the particle size distribution of emulsions were determined using a static light scattering equipment called Mastersizer 3000 (Malvern Instruments Ltd, Malvern, UK).

\subsection{Scanning electron microscopy}

Morphological features of butter oil emulsions were examined using scanning electron microscopy (SEM) (FEI, Quanta). Samples were studied in a) fluid state using environmental attachment (at $15^{\circ} \mathrm{C}$ ), b) freeze-dried (Scanvac, Coolsafe) form (at 8 ${ }^{\circ} \mathrm{C}$ ), and c) in vacuum dried form (at $8{ }^{\circ} \mathrm{C}$ ) with high-vacuum option in SEM. The latter two were gold-sputtered for 35 seconds.

\section{Results and discussion}

\subsection{Self-stabilized emulsions of butter oil}

Among the range of butter oil emulsions (5\%,10\%, 15\% and 20\%) prepared, only 5\% emulsions were stable for more than $30 \mathrm{~min}$ whereas others undergo rapid phase separation (Fig. 2). There are some fundamental reasons behind the instability of these self-stabilized butter oil emulsions (self-stabilization: butter oil acting as an emulsion stabilizer). First, the butter oil is composed of more than $99 \%$ lipids, which usually have a limited solubility in water (Newton, 2014). Second, there is about two orders of magnitude difference in their viscosities (viscosity of the butter oil is $\sim 45 \mathrm{mPa}$.s 
(Bürkle GmbH, 2011) while the viscosity of water equals to $0.65 \mathrm{mPa} . \mathrm{s}$ (Korson et al., 1969) measured at $40^{\circ} \mathrm{C}$ ). Moreover, some of the lipids in butter oil start solidifying when cooled below $40{ }^{\circ} \mathrm{C}$ promoting further phase separation (Deshpande and Kulkarni, 2012; Sulieman et al., 2013). These features hinder the self-stabilization of butter oil emulsions. Due to its low density $\left(\sim 0.93 \mathrm{gm} / \mathrm{cm}^{3}\right.$ (Deshpande and Kulkarni, 2012; Sulieman et al., 2013), the butter oil floats on the water as shown in Fig. 2 (indicated by block arrows). In addition, the local phase separation contributes towards formation of oily droplets. Such droplets were (partly) visible in high butter oil emulsions giving them the yellow tinge (Fig. 2).

\subsection{Kinetic stabilization of butter oil emulsions}

Some of the aforementioned problems, causing emulsions' destabilization, can be overcome by using stabilizer molecules ((Garti et al., 1993; Kulkarni and Glatter, 2012; Sjoblom, 2005). The process is called 'kinetic stabilization', where the emulsion is kept stable over time thereby significantly decreasing common destabilization parameters such as droplet coalescence, creaming or Ostwald ripening (Sjoblom, 2005). Sterically modulated physicochemical forces between the droplet interfaces create a barrier preventing their aggregation, thus enhancing kinetic stability of an emulsion. We used a (food grade) non-ionic tri-block co-polymer pluronic $®$ F127 $(\mathrm{MW}=12.6 \mathrm{kDa})$ to stabilize butter oil emulsions. This is an amphiphilic molecule formed of two blocks of (PEO) ${ }_{99}$ and one block of (PPO) 67 imparting hydrophilic and hydrophobic characters to the molecule, respectively (Desai et al., 2001). The hydrophobic block interacts with the hydrophobic chains of lipid molecules that usually self-assemble into inverse (type 2) (Kulkarni et al., 2011b) micelles, while the 
hydrophilic blocks face towards aqueous medium thereby enhancing the dispersibility of oil droplets/particles (Fig. 3a).

The viscosity of the kinetically stabilized emulsions was found to increase virtually once they cool down after sonication as well as with increasing the butter oil concentration. The consistency imparted due to the viscosity difference ranged from water-like to creamy, in nature. This can be attributed to the solidification of lipids, whose melting point lies around $37^{\circ} \mathrm{C}$ (Deshpande and Kulkarni, 2012; Sulieman et al., 2013). Despite of this, stable and reproducible emulsions could be prepared up to $15 \%$ of the butter oil (Fig. $3 b$, c, d) while the $20 \%$ sample struggled to stay stable after about an hour (Fig. 3e). Nevertheless, the separated oil in the latter was completely redispersed upon gentle shaking (explained later in Fig. 7).

\subsection{Nano-micro structural characterization of butter oil emulsions}

Small angle X-ray scattering was used to determine the type of phase (nanostructure) exhibited by butter oil lipids. At $25^{\circ} \mathrm{C}$, undispered butter oil showed lamellar nanostructure in presence of water, as evidenced from the typical peaks in Fig. 4 (shown by block arrows); the d-spacing was found to be $41.5 \pm 0.5 \AA$. Two little peaks (Fig. 4, shown by stars) were observed for kinetically stabilized emulsion which may mean that the partial order is still retained inside the stabilized particles. Typical upturn in the low $q$ region with scattering intensity $I(q) \propto q^{-2}$ indicate the formation of particulate structures (Fig. 4) (Pearce et al., 2014). However, there was no characteristic signal seen in self-stabilized emulsions, presumably depicting the partial aggregation. This was also supported by the observation of rather wide particle size distribution (studied by Mastersizer) in self-stabilized emulsions (Fig. 5). Kinetically 
stabilized emulsions, on the other hand, exhibited sub-micron sized particles with relatively narrow size distribution (Fig. 5). Particle size and microstructure of kinetically stabilized emulsions was also confirmed with SEM micrographs as shown in Fig. 6.

\subsection{Stability and shelf-life of butter oil emulsions}

As mentioned earlier, 5\% emulsions were stable with and without stabilizer. Butter oil in higher concentrations, however, became separated within an hour in case of the emulsions prepared without stabilizer (see Fig. 2). Kinetic stabilization (using stabilizer) improved the stability of butter oil emulsions up to about a month. After which these emulsions looked as shown in Fig. 7a where some of the butter was seen to be forming a layer on the top (indicated by arrows). The thickness of the layer increased with the concentration of butter oil. Nevertheless, the separation was significantly less as compared to the separation in Fig. 2, which is evident from the color of the separated layer (dark yellow in Fig. 2 while pale yellow in Fig. 7a). More importantly, all of these emulsions could be made homogeneous again upon gentle shaking (2-3 upside down moves), as shown in Fig. 7b. Emulsions were stable, in this manner, for at least studied six months. The shelf-life of these emulsions was observed for about six months (at room temperature) during which there was no characteristic odor or growth of fungus demonstrating the degradation of the butter oil; for instance, similar to the Mendez-Vilas report (Méndez-Vilas, 2012). 


\section{Conclusions and Perspectives}

Oil-in-water emulsions, from high lipid ( $>99 \%$ ) containing butter oil, were prepared using an ultrasonic processing technology. Being itself a food material, the butter oil clearly exhibits biocompatibility and food grade properties, therefore the emulsions reported here are highly suited for potential biotechnological applications. Butter oil emulsions provide a number of advantages as compared to the bulk butter oil; for instance, the flavour (sometimes unwanted) generated during the preparation of butter oil via Maillard reaction (Newton, 2014; Newton et al., 2015) is significantly reduced upon its emulsification. These emulsions contain a very high $(80 \%$ to $>95 \%)$ concentrations of water thereby improving their applicability in formulation sector by enabling the use of aqueous phase to solubilize hydrophilic molecules along with the lipophilic ones - solubilized in the cores of lipid particles (Kulkarni and Glatter, 2012). In addition, these emulsions have a range of (fluid to creamy) consistencies which can be finely controlled with the lipid contents. Further characteristics, including long term stability, extended shelf life, submicron sized particulate form and variable viscosity, facilitate the butter oil emulsions to be exploited for various pharmaceutical and cosmetic formulations. As compared to the lipids that are commonly used for preparing poorly soluble drug formulations, the butter oil lipids are rather cheap and easily available. Therefore, butter oil emulsions find great potential for formulation based applications. To summarize, the reported butter oil emulsions: 1) retain hydrophobic properties of the original butter oil at the cores of dispersed particles, 2) overcome a problem of butter oil-water immissibility, 3) display fluid consistency, 4) exhibit enormous interfacial area as well as aqueous region, 5) are easy to prepare reliably, and 6) are stable for several months. These properties are highly suitable for developing food, pharmaceutical and cosmetic formulations. 


\section{Acknowledgements}

We would like to thank Ms. Sana Sayed for the experimental work on butter oil emulsions, while Prof Michael Rappolt and Dr Amin Sadeghpour from the_School of Food Science \& Nutrition, University of Leeds, Leeds, UK for allowing us to use SAXS and Mastersizer facilities. Also thanks to Dr Yogita Patil-Sen who and Amin performed experiments on above facilities. We kindly acknowledge Ms. Priti C. Kulkarni for assisting us with the preparation of butter oil.

\section{References}

Acharya, K.T. 1997. Ghee, vanaspati, and special fats in India. In: Lipid Technologies and Applications, (F.D. Gunstone, F.B. Padley, eds.), Taylor \& Francis.

Bishwas, N.C. 2013. Ointment for treatment of wound. World Patent, WO2013021396 A1.

Chong, J.Y.T., Mulet, X., Boyd, B.J., Drummond, C.J. 2015. Chapter Five - Steric Stabilizers for Cubic Phase Lyotropic Liquid Crystal Nanodispersions (Cubosomes). In: Advances in Planar Lipid Bilayers and Liposomes, (C.V.K. Aleš Iglič, R. Michael, eds.) pp. 131-187, Academic Press.

Cope, R.B., Bosnic, M., Boehm-Wilcox, C., Mohr, D., Reeve, V.E. 1996. Dietary Butter Protects against Ultraviolet Radiation-Induced Suppression of Contact Hypersensitivity in Skh:HR-1 Hairless Mice. J. Nutr. 126, 681-692.

Desai, P.R., Jain, N.J., Sharma, R.K., Bahadur, P. 2001. Effect of additives on the micellization of PEO/PPO/PEO block copolymer F127 in aqueous solution. Colloids Surf., A. 178, 57-69.

Deshpande, P., Kulkarni, K. 2012. Production and evaluation of biodiesel from palm oil and ghee (clarified butter). Chemical and Process Engineering Research. 2, 33-42. 
Engelskirchen, S., Maurer, R., Glatter, O. 2011. Effect of glycerol addition on the internal structure and thermal stability of hexosomes prepared from phytantriol. Colloids Surf., A. 391, 95-100.

Fatouh, A.E., Singh, R.K., Koehler, P.E., Mahran, G.A., El-Ghandour, M.A., Metwally, A.E. 2003. Chemical and thermal characteristics of buffalo butter oil fractions obtained by multi-step dry fractionation. Lebensmittel-Wissenschaft Und-Technologie-Food Science and Technology. 36, 483-496.

Garti, N., Reichman, D., Hendrickx, H.A.C.M., Dickinson, E., Jackson, L.K., Bergenstahl, B. 1993. Hydrocolloids as Food Emulsifiers and Stabilizers. Food Struct. 12, 411-426.

Heyn, B. 1987. Ayurvedic medicine: the gentle strength of Indian healing. Thorsons Publishers.

Korson, L., Drost-Hansen, W., Millero, F.J. 1969. Viscosity of water at various temperatures. J. Phys. Chem. 73, 34-39.

Kulkarni, C.V., Glatter, O. 2012. Hierarchically Organized Systems Based on Liquid Crystalline Phases. In: Self-Assembled Supramolecular Architectures: Lyotropic Liquid Crystals, (N. Garti, ed.), John Wiley \& Sons, Inc.

Kulkarni, C.V., Moinuddin, Z., Patil-Sen, Y., Littlefield, R., Hood, M. 2015. Lipid-hydrogel films for sustained drug release. Int. J. Pharm. 479, 416-421.

Kulkarni, C.V., Tomšič, M., Glatter, O. 2011a. Immobilization of Nanostructured Lipid Particles in Polysaccharide Films. Langmuir. 27, 9541-9550.

Kulkarni, C.V., Wachter, W., Iglesias, G.R., Engelskirchen, S., Ahualli, S. 2011b. Monoolein: A Magic Lipid? Phys Chem Chem Phys. 13, 3004-3021.

Lad, V. 1999. The Complete Book of Ayurvedic Home Remedies. Three Rivers Press.

Liu, L.Y. 2014. Medicinal liquor capable of tonifying lung and keeping young. Chinese Patent CN103849527 A. 
Méndez-Vilas, A. 2012. Microbes in Applied Research: Current Advances and Challenges. Hackensack, NJ.

Nakano, M., Teshigawara, T., Sugita, A., Leesajakul, W., Taniguchi, A., Kamo, T., Matsuoka, H., Handa, T. 2002. Dispersions of Liquid Crystalline Phases of the Monoolein/Oleic Acid/Pluronic F127 System. Langmuir. 18, 9283-9288.

Newton, A. 2014. Flavour formation in ghee. In: Biology, University of Canterbury.

Newton, A.E., Fairbanks, A.J., Golding, M., Andrewes, P., Gerrard, J.A. 2015. The influence of emulsion structure on the Maillard reaction of ghee. Food Chem. 173, 1243-1249.

Pariza, M.W. 1991. A new cancer inhibitor in dairy products. Bull. Int. Dairy Fed. 257 2930.

Patil-Sen, Y., Sadeghpour, A., Rappolt, M., Kulkarni, C.V. 2016. Facile Preparation of Internally Self-assembled Lipid Particles Stabilized by Carbon Nanotubes. J. Vis. Exp. e53489.

Pearce, T.R., Waybrant, B., Kokkoli, E. 2014. The role of spacers on the self-assembly of DNA aptamer-amphiphiles into micelles and nanotapes. Chem Comm. 50, 210-212.

Sangwan, R.B., Udaikumar, G., Malik, R.C. 2000. Stability of emulsions of sodium caseinate and ghee as influenced by temperature. Indian J. Dairy Biosci. 11, 134-135.

Saravanan, A.N., Suchitra, N., Dhandayuthapani, K. 2007. Role of saturated fatty acids in lipase production - using Pseudomonas Aeruginosa. J. Food Biochem. 31, 748-756.

Shah, E.M. 2014. Base material for pharmaceutical and/or cosmetic cream (herbal composition for itchy or infected skin). US Patent US8703212 B2.

Sharma, H.M. 1990. Butter oil (ghee) - Myths and facts. Indian J Clin Pract 1, 31.

Sjoblom, J. 2005. Emulsions and Emulsion Stability: Surfactant Science Series/61. CRC Press. 
Sserunjogiab, M.L., Abrahamsena, R.K., Narvhusa, J. 1998. A Review Paper: Current Knowledge of Ghee and Related Products. Int. Dairy Journal. 8, 677-688.

Sujatha, K., Sarashetti, R.S. 2015. Vision and Ghee. JBSO. 3, 143-146.

Sulieman, A.M.E., Mohammed, M.B., Ali, A.O. 2013. Physicochemical and Sensory Properties of Traditionally and Laboratory Made Ghee (Samin) of the Sudan. Int. J. Food Sci. Nutr. Engg. 3, 7-11.

The Culinary Institute of America, Donovan, M.D. 1995. The New Professional Chef. John Wiley \& Sons.

Tirtha, S.S. 1998. The Ayurveda Encyclopedia. Ayurveda Holistic Center Press, Bayville, NY.

USDA. 2016. United States Department of Agriculture (USDA) National Nutrient Database for Standard Reference Release 28

Yaghmur, A., de Campo, L., Sagalowicz, L., Leser, M.E., Glatter, O. 2005. Emulsified Microemulsions and Oil-Containing Liquid Crystalline Phases. Langmuir. 21, 569577. 
Fig. 1 Main constituents of butter (a) and butter oil (c) shown in percent weights; the data obtained from USDA, National Nutrient Database for Standard Reference Release 28. (USDA, 2016). Schematic of the preparation of butter oil from butter (b); images courtesy Priti C. Kulkarni. $20 \times 6 \mathrm{~mm}(300 \times 300 \mathrm{DPI})$ 
Fig. 2 Butter oil was able to self-stabilize its emulsion at low concentration, that is $5 \%$ as shown in a) while it was phase separated in all higher concentrations as shown by arrows in b), c) and d) respectively for 10, 15 and $20 \%$ butter oil emulsions. Images were captured after 30 min of their preparation. $20 \times 7 \mathrm{~mm}(300 \times 300 \mathrm{DPI})$ 
Fig. 3 a) Schematic drawing depicts the role of stabilizer (F127) in dispersing the oil droplets/particles in o/w emulsion. Kinetically stabilized o/w emulsions with 5,10,15 and 20\% butter oil are shown in b), c) d) and e) respectively. Emulsions were stable up to $15 \%$ butter oil content, while $20 \%$ butter oil emulsion started phase separating after about an hour. $16 \times 3 \mathrm{~mm}$ (300 x 300 DPI) 


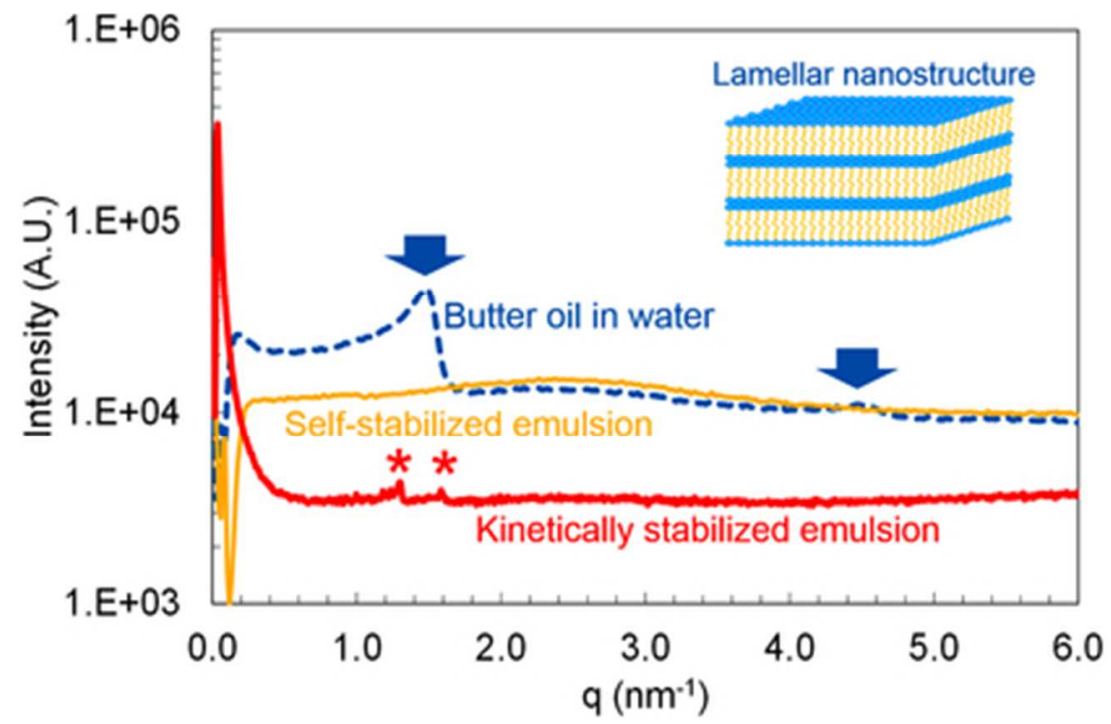

Fig. 4 Small angle X-ray scattering studies revealed the presence of lamellar nanostructure (peaks indicated by arrows) in the hydrated butter oil sample while no peak was observed for self-stabilized emulsions. Kinetically stabilized particles showed typical upturn in low q region with tiny peaks (shown by $*$ ) located around the first order peak of the lamellar nanostructure of the hydrated butter oil sample. $17 \times 11 \mathrm{~mm}(600 \times 600$ DPI) 


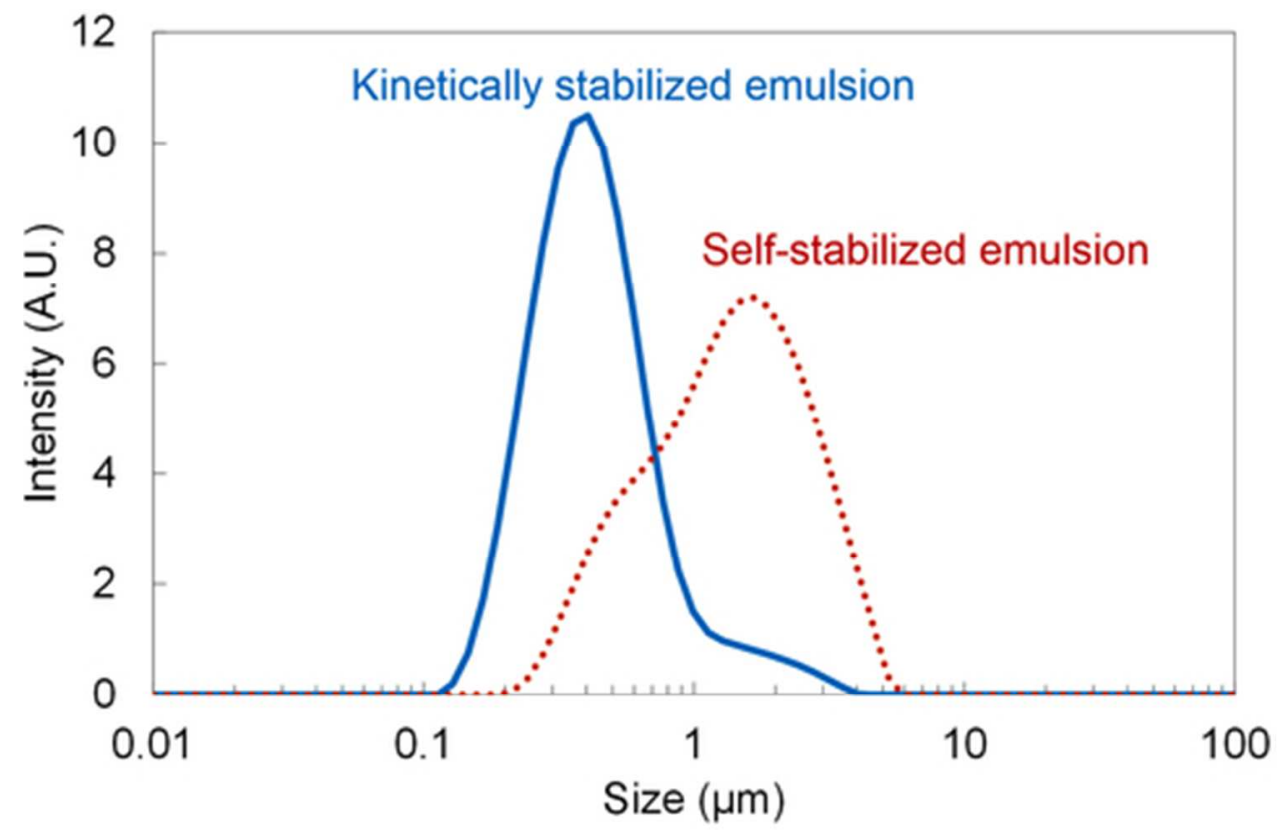

Fig. 5 Particle size analysis elucidates the narrow size distribution in case of kinetically stabilized emulsions as compared to the self-stabilized emulsion. Emulsions used for performing SAXS and particle size measurements contained $5 \%$ butter oil. $20 \times 13 \mathrm{~mm}(600 \times 600 \mathrm{DPI})$ 

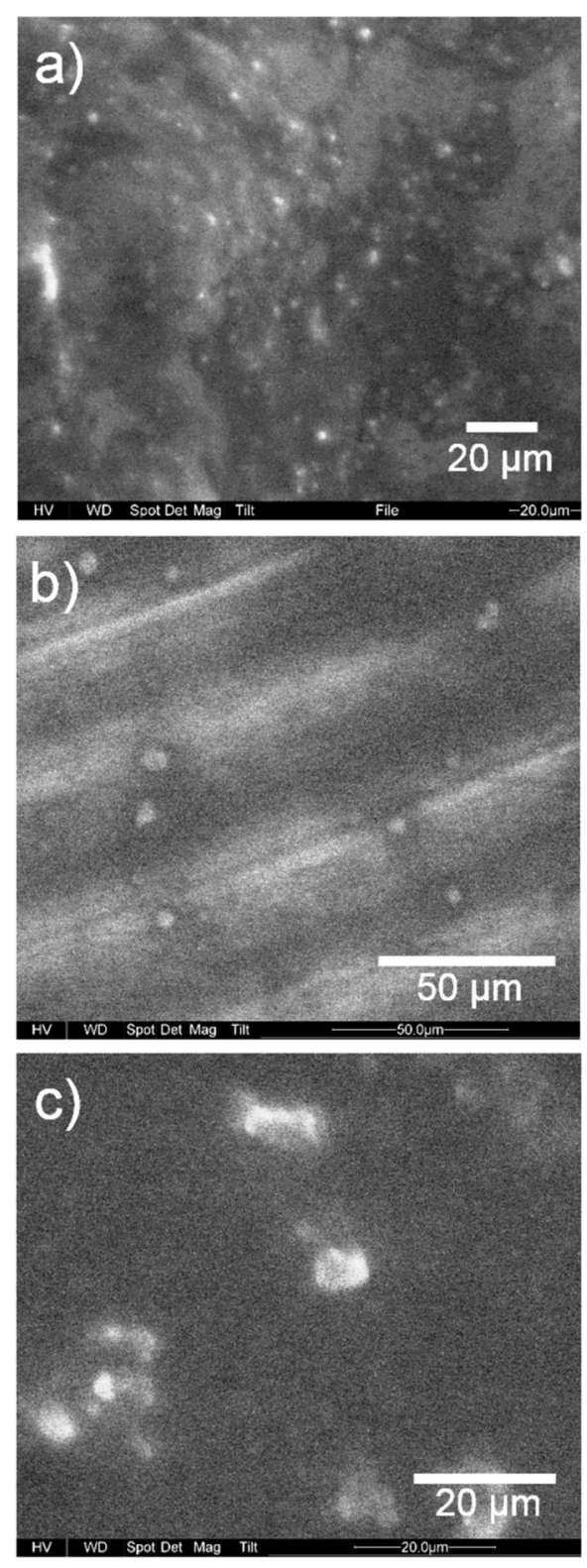

Fig. 6 Scanning electron microscopy (SEM) micrographs of butter oil emulsions in a) fluid state, b) freezedried form and c) high vacuum mode in SEM. The particle size in the micron range is evident from all of these images.

$57 \times 156 \mathrm{~mm}(300 \times 300 \mathrm{DPI})$ 
Fig. 7 Emulsions with high concentration of butter oil ( $\geq 10 \%)$ partially destabilized after about a month as shown in a), which were homogenised again after gentle shaking as in b). Arrows indicate the butter oil layer formed in the upper part of emulsions. Re-dispersion of the emulsions (by gentle shaking) could be effectively done for at least six months (of study time). $40 \times 34 \mathrm{~mm}(300 \times 300$ DPI $)$ 